\title{
Chapitre 2 : La protéine prion cellulaire (PrPc) : propriétés et rôle physiologique
}

Les propriétés et le rôle physiologique de la protéine prion sont encore mal connus, de même que la façon et les raisons pour lesquelles la protéine prion normale est transformée en protéine prion pathogène. Ces différentes questions sont importantes, à la fois pour comprendre les mécanismes moléculaires à l'origine des encéphalopathies spongiformes transmissibles et pour rechercher des possibilités de thérapie. C'est la raison pour laquelle divers types de travaux sont conduits sur ces questions, utilisant différentes approches.

L'article de Lepage et al est basé sur l'approche physicochimique de la structure de la protéine prion. L'objectif de ces travaux est de rechercher les domaines de la protéine prion qui pourraient avoir un rôle clé dans la transconformation de la protéine normale en protéine pathogène, tant pour induire que pour réguler ce processus.

L'article de H. Rezaei et al est basé sur une approche physico-chimique et structurale de la protéine prion visant à comprendre la relation entre le polymorphisme génétique de cette protéine et l'état de résistance ou de sensibilité à la tremblante. Les données devraient permettre l'établissement d'un lien entre les propriétés structurales et le développement de la pathologie.

L'article de Moudjou et al est basé sur une approche immunochimique. Des anticorps monoclonaux ont été mis au point, avec des affinités particulières pour certaines régions de la protéine prion PrP. Ainsi certains anticorps reconnaissent différentes formes glycosylées de la protéine et discriminent les allèles de la PrP associés à la susceptibilité des moutons à la tremblante. Ces réactifs offrent des perspectives dans plusieurs domaines, notamment dans l'étude du rôle de la glycosylation dans la capacité de la protéine prion à être convertie en protéine pathogène et dans l'analyse fine des profils glycotypiques de la protéine anormale, dans le cadre du typage moléculaire des souches de prions. Ces anticorps peuvent également être utilisés comme moyen de génotypage rapide des moutons.

Enfin, l'article de Gatti et al analyse le rôle physiologique de la protéine prion cellulaire. Des travaux ont été entrepris pour établir la présence et les voies de sécrétion de la protéine prion normale dans différents organes et tissus de l'organisme, ainsi que son rôle, en utilisant différents modèles animaux et cellulaires. 